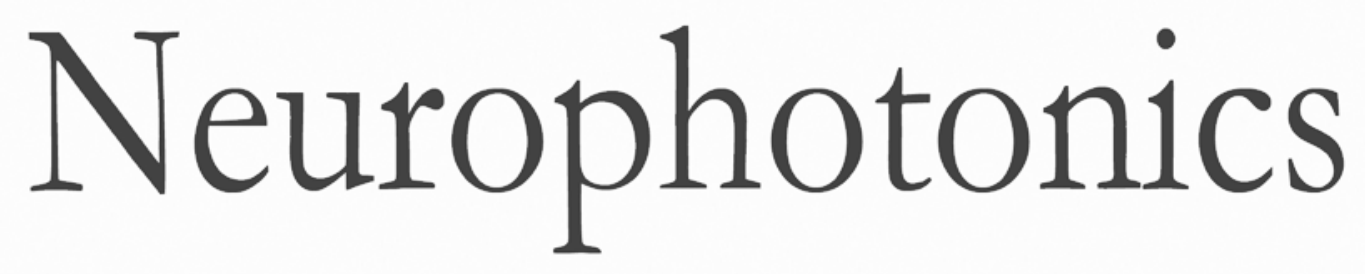

Neurophotonics.SPIEDigitalLibrary.org

\title{
NPH 2018 Reviewer List
}


Neurophotonics would like to sincerely thank the following individuals who served as reviewers in 2018. The success of our publication hinges on the voluntary contributions of time and energy put forth by these professionals.

\begin{tabular}{|c|c|c|}
\hline Ardalan Aarabi & Xiaosu Hu & Jun Qian \\
\hline Yama Akbari & Theodore Huppert & Daniel Razansky \\
\hline Srdjan Antic & Sahar Jahani & Allan Reiss \\
\hline Misa Arizono & Jana Kainerstorfer & Nicolas Renier \\
\hline Hasan Ayaz & Emanuela Keller & Herve Rigneault \\
\hline Wesley Baker & Ramin Khatami & Darren Roblyer \\
\hline Ken Berglund & Beop-Min Kim & Nadege Roche-Labarbe \\
\hline Karla Bergonzi & Bernd Kuhn & Angelika Rueck \\
\hline Thomas Blanpied & Stefania Lancia & Christian Rummel \\
\hline Robert Bonin & Kang Lee & Farzad Salehpour \\
\hline Heather Bortfeld & Frederic Lesage & Hiroki Sato \\
\hline Ralf Brinkmann & Jun Li & Daniel Schmidt \\
\hline Erin Buckley & Lei Li & Felix Scholkmann \\
\hline Robert Campbell & Antonia Lichtenegger & Kenneth Shepard \\
\hline Stefan Carp & Adam Liebert & Andy Shih \\
\hline Bernard Choi & Chao Liu & Ludovico Silvestri \\
\hline Euiheon Chung & Heiko Luhmann & Roma Siugzdaite \\
\hline Adam Cohen & Hongtao Ma & Zachary Smith \\
\hline Robert Cooper & Avraham Mayevsky & Marcelo Sousa \\
\hline Irene Costantini & Masahito Mihara & Vivek Srinivasan \\
\hline Marek Czosnyka & Erika Molteni & Herbert Stepp \\
\hline Helga De Oliveira Miguel & Yusuke Moriguchi & Colin Sullender \\
\hline Patrick Drew & Theresa Moulton & Smrithi Sunil \\
\hline Mathieu Ducros & Hideji Murakoshi & Ilias Tachtsidis \\
\hline Adam Eggebrecht & Timothy Murphy & Gentaro Taga \\
\hline Valentina Emiliani & M. Nasiriavanaki & Toshimitsu Takahashi \\
\hline Tomer Fekete & Charles Nelson & Fenghua Tian \\
\hline Alissa Ferry & Jon Newman & Yunjie Tong \\
\hline Rodrigo Forti & Liming Nie & Alessandro Torricelli \\
\hline Amanda Foust & Sergio Novi & Snow Tseng \\
\hline Blaise Frederick & Jonathan Nylk & Daisuke Tsuzuki \\
\hline Peter Freestone & Felipe Orihuela-Espina & Vassiliy Tsytsarev \\
\hline Martin Graf & Annette Pantall & Feng Wang \\
\hline Edgar Guevara & Ashwin Parthasarathy & Hui Wang \\
\hline Pengfei Hai & Katherine Perdue & Toru Yamada \\
\hline Mahlega Hassanpour & T. Pfefer & Meryem Yucel \\
\hline Masahiro Hirai & Kaspar Podgorski & Han Zhang \\
\hline Fumitaka Homae & Eric Potma & Wen Zhang \\
\hline Keum-Shik Hong & Philippe Pouliot & \\
\hline
\end{tabular}

